\title{
Predicción de resultados académicos con la aplicación nntool en Matlab utilizando redes neuronales artificiales
}

\section{Prediction of academic results with the nntool application in Matlab using artificial neural networks}

Dulce Lucero Capuñay Sanchez, ${ }^{11}$ Fernando Alain Incio Flores, ${ }^{2}$ Ronald Omar Estela Urbina, ${ }^{3}$ Luis Montenegro Camacho, ${ }^{4}$ Jorge Antonio Delgado Soto, ${ }^{5}$ Johnny Cueva Valdivia ${ }^{6}$

\author{
Universidad Cesar Vallejo, Lima, Perú ${ }^{14}$ \\ Universidad Nacional Intercultural Fabiola Salazar Leguía de Bagua, Perú ${ }^{23}$ \\ Universidad Nacional de Cajamarca, Cajamarca, Perú ${ }^{5}$ \\ Universidad Nacional Santiago Antúnez de Mayolo, Ancash, Perú ${ }^{6}$

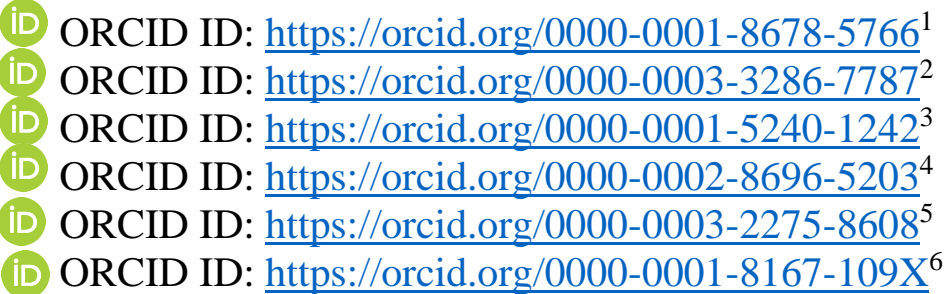

Recibido: 20 de junio de 2021

Aceptado: 15 de octubre de 2021

\section{Resumen}

En el 2018, Perú participó de la Evaluación Internacional de Estudiantes (PISA), donde se evidenciaron resultados desalentadores en los estudiantes de educación básica regular. En tal sentido, se consideró la predicción de resultados académicos como un instrumento de mejora del rendimiento escolar. El objetivo de esta investigación fue predecir el promedio anual de estudiantes del segundo grado de la Institución Educativa $\mathrm{N}^{\circ} 16093$ en la provincia de JaénPerú, mediante el diseño e implementación de una red neuronal artificial (RNA). Para la recolección de datos de las variables que influyen en el promedio anual del estudiante, se elaboró un cuestionario de respuestas dicotómicas. En la validación y confiabilidad, se utilizó el criterio de juicio de expertos y la prueba Kuder-Richarson respectivamente, en el cual el coeficiente de confiabilidad obtenido en una prueba piloto aplicado a 15 estudiantes fue de 0.8359. En el software científico Matlab con la ayuda de la aplicación nntool, se diseñó la RNA formada por tres capas ocultas y una capa de salida. La RNA durante el entrenamiento, la validación y la prueba, registró un coeficiente de correlación ponderado de 0.967190 , y un error cuadrático medio de 0.05011 . El modelo neuronal implementado bajo las condiciones dadas logró una efectividad del $88.670 \%$ y $98.522 \%$. 
Palabras clave: Rendimiento académico, nntool, redes neuronales artificiales, predicción, educación.

\begin{abstract}
In 2018, Peru participated in the International Student Assessment (PISA), where disappointing results were evidenced in students of regular basic education. In this sense, the prediction of academic results was considered as an instrument for improving school performance. The objective of this research was to predict the annual average of second grade students of the Educational Institution $\mathrm{N}^{\circ} 16093$ in the province of Jaén-Peru, through the design and implementation of an artificial neural network (ANN). To collect data on variables that influence the annual average of the student, a questionnaire with dichotomous responses was developed. In the validation and reliability, we used the expert judgment criterion and the Kuder-Richarson test respectively, the reliability coefficient obtained in a pilot test applied to 15 students was 0.8359. In the Matlab Scientific Software with the help of the nntool application, the RNA was designed consisting of three hidden layers and an output layer. The ANN during training, validation and testing, registered a weighted correlation coefficient of 0.967190 , and a mean square error of 0.05011 . The neural model implemented under the given conditions achieved an effectiveness of $88.670 \%$ and $98.522 \%$.
\end{abstract}

Keywords: Academic performance, nntool, artificial neural networks, prediction, education.

\title{
Introducción
}

Predecir los resultados académicos de los estudiantes de manera temprana, permite al docente, tomar las medidas correctivas y necesarias en la búsqueda de estrategias a fin de mejorar el logro de competencias en sus estudiantes. En el Perú, el actual diseño curricular nacional que el Ministerio de Educación ha elaborado, está fundamentado en el logro de competencias. Una competencia es la destreza que adquiere el estudiante para combinar conocimientos, actitudes, aptitudes y habilidades para dar solución a una situación problemática en un tiempo determinado (Echeverría-Ramírez \& Mazzitelli, 2021; Hernández, 2018; Incio \& Capuñay, 2020; Olascoaga, 2020; Sagredo et al., 2021). Bajo este concepto, se entiende al rendimiento académico, como el sistema que mide el logro de competencias en una escala vigesimal, expresada en el promedio final del estudiante en determinadas materias académicas durante un periodo de estudios (Comella et al., 2021; Criollo et al., 2017; González-García et al., 2019; González-Velasco et al., 2021; Rodríguez et al., 2021). Por ello, la formación académica del estudiante de educación básica, es consecuencia de la combinación de una serie de factores de carácter endógeno y exógeno que lo rodea, diversos autores agrupan a estos agentes influyentes del rendimiento académico de acuerdo a sus funcionalidades: de orden personal, social e institucional (Ferraces et al., 2021; Pizarro et al., 2021; Porras \& Ortega, 2021). 
Por otro lado, una RNA es un modelo matemático y computacional, diseñado para aprender mediante el entrenamiento, valiéndose de un conjunto de datos de entrada que le permiten su aprendizaje a través un conjunto de neuronas artificiales interconectadas entre sí, por medio de funciones matemáticas de activación, el cual simula el comportamiento del cerebro humano: aprender mediante la experiencia (Barreto \& Picón, 2020; da Silva et al., 2020; Goyes-Peñafiel \& Hernandez-Rojas, 2021; Pal \& Chakrabarty, 2020; Şenyurt et al., 2020). Entre los tipos de redes neuronales artificiales más conocidas tenemos al Perceptrón simple y Perceptron multicapa, Adaline, Redes de Feed-Forward, Redes de Elman, Redes de Hopfield. En esta investigación, y de acuerdo a la naturaleza de los datos, se ha utilizado las Redes de Feed-Forward, las cuales se caracterizan por la flexibilidad en la implementación en el número de capas ocultas y el número de neuronas en cada capa según los resultados en cada prueba. Diversas investigaciones sustentan que las Redes de Feed-Forward superan las limitaciones que presentan los tipos de redes neuronales anteriormente mencionadas en cuanto a su estructura y procesamiento de la data (Figueroa-Garcia et al., 2021; Incio et al., 2021; H. E. D. Rodríguez et al., 2021; Sosa et al., 2021; Wingerter et al., 2020).

Las investigaciones destacan la importancia de las redes neuronales artificiales en la predicción en el campo de la educación; en el contexto internacional, Martins et al. (2020) realizaron una investigación para predecir la deserción estudiantil, apoyándose en algoritmos de minería de datos como árboles de decisión y redes neuronales artificiales; lograron implementar modelos neuronales para predecir la deserción escolar universitaria, logrando de esta manera que los docentes responsables de cada asignatura adopten medidas y decisiones estratégicas para reducir la tasa de abandono de estudios al culminar el periodo académico (Costa et al., 2017). En este caso, Lejía et al. (2010) realizaron una investigación para predecir el rendimiento en matemáticas de estudiantes pre universitarios, para lograr su objetivo trabajaron redes neuronales con algoritmos de retro propagación y regresión generalizada, mediante una validación cruzada de 10 veces logró identificar que el mejor modelo de predicción es el implementado con el algoritmo de retro propagación con una efectividad de precisión del $66.67 \%$ y $71.11 \%$.

En Turquía Çetinkaya y Baykan (2020) en su investigación basada en redes neuronales artificiales implementaron un modelo neuronal para predecir el talento en programación en estudiantes de secundaria, la RNA fue implementada en Matlab con la aplicación nnstart mediante regularización bayesiana, gradiente conjugado y el algoritmo Levenberg Marquardt, tomando datos históricos y apoyado en un curso implementado en la plataforma Code.org logró predecir y comparar la efectividad en la predicción, el modelo neuronal implementado con 
mayor efectividad fue mediante el algoritmo Levenberg Marquardt. En la República de Cuba, Álvarez et al. (2016) diseñaron e implementaron un modelo de RNA con una capa de entrada oculta y de salida para predecir el rendimiento académico de estudiantes de educación superior en los curso de Estructuras de Datos I y II, el modelo implementado en Matlab logró una efectividad de predicción del 78\% y $75 \%$ respectivamente en cada curso.

En el contexto nacional en la Universidad Nacional Intercultural Fabiola Salazar Leguía de Bagua, Incio et al. (2021) realizaron una investigación para predecir el rendimiento académico en estudiantes universitarios de la carrera de Ingeniería Civil en el curso de Física, aprovechando los algoritmos implementados en la Toolbox de Matlab: Scaled Conjugate Gradien y Levenberg Marquardt, implementaron dos redes neuronales con una capa de entrada, una capa oculta y una capa de salida, los resultados muestran que el algoritmo que logró mayor efectividad en el predicción fue el Levenberg Marquardt (86\%), mientras que el Scaled Conjugate Gradien (70\%). En la Universidad Agraria la Molina, Menacho (2017) empleando diagramas de árbol de decisión, redes neuronales y redes bayesianas logró implementar un modelo neuronal con una efectividad del $71 \%$ para predecir los resultados académicos del curso de Estadística General.

Por tanto, el objetivo de la investigación fue diseñar e implementar un modelo de red neuronal artificial mediante la aplicación nntool en el software científico Matlab, a fin de predecir el rendimiento académico de los estudiantes del segundo grado de la Institución Educativa N 16093 en la provincia de Jaén-Perú.

\section{Materiales y métodos}

En esta investigación se pretendió recolectar la percepción de todos los estudiantes del tercer, cuarto y quinto grado de educación secundaria de la Institución Educativa $\mathrm{N}^{\circ} 16093$ con respecto a las variables que influyen en el rendimiento académico, siendo en su totalidad 235 estudiantes; sin embargo-y debido a la coyuntura actual por la que atraviesa la educación virtual en Perú como consecuencia del Covid-19 y los múltiples obstáculos que esta presenta, entre ellos la conectividad - solamente se ha logrado recolectar datos de 203 estudiantes. Los datos recolectados en esta investigación están relacionados con los factores de índole personal, social e institucional que influyen significativamente en el rendimiento académico del estudiante de secundaria, para la elaboración del instrumento de recolección de datos (cuestionario) se realizó un trabajo previo de revisión bibliográfica (Ferraces et al., 2021; Pizarro et al., 2021; Ramos \& Gómez, 2021), luego se elaboró el cuestionario con 45 preguntas de respuestas dicotómicas; las preguntas del 1-19 están referidas a los factores personales, del 
20-30 a los factores sociales y del 31-45 a factores institucionales, para la validación del instrumento se empleó el criterio de juicio de expertos y para la confiabilidad la prueba KuderRichardson $\left(\mathrm{KR}_{20}\right)$ aplicado a un piloto de 15 estudiantes (Díaz-Muñoz, 2020), siendo el coeficiente de confiabilidad obtenido de 0.8359 el cual es calificado como bueno (Foster, 2020, 2021).

El mencionado cuestionario fue elaborado con la aplicación Google Forms y enviado a los estudiantes por medio de un enlace WhatsApp, correo electrónico o en su defecto mediante llamada telefónica, garantizando en todo momento la confidencialidad de la información brindada por los estudiantes encuestados, el respeto estricto a la normativa de la institución educativa y la no manipulación de la data obtenida. Una vez aplicado el cuestionario, se descargó un archivo Excel generado por la aplicación conteniendo la percepción de los estudiantes respecto a las variables que influyen en el rendimiento académico. Asimismo, se utilizó el diseño metodológico CRISP-DM, según International Business Machines Corporation (2021) es el modelo que mejor se adapta a investigaciones que involucran minería de datos y redes neuronales artificiales.

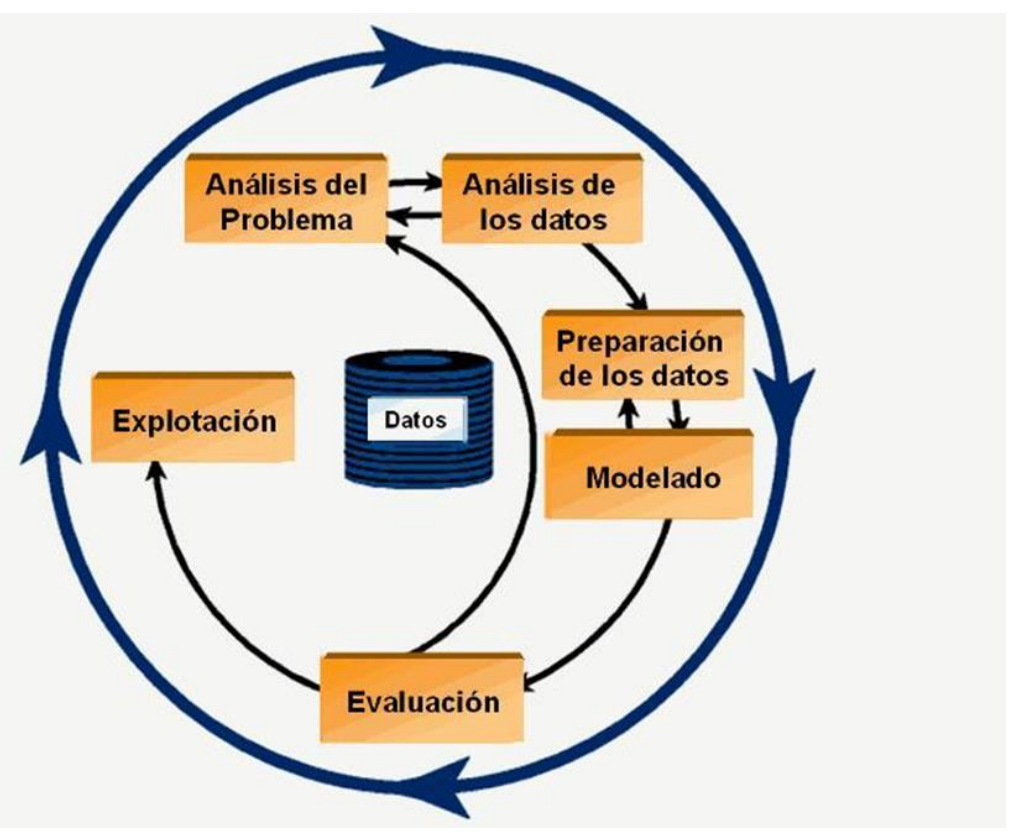

Figura 1. International Business Machines Corporation

La evaluación del modelo de red neuronal implementado en Matlab 2016a se realizó teniendo en cuenta el coeficiente de correlación (R) y el error cuadrático medio (MSE, por sus siglas en Ingles). 


$$
R^{2}=1-\frac{\sum_{i=1}^{n}\left(y_{i c}-y_{i m}\right)^{2}}{\sum_{i=1}^{n}\left(y_{i c}-y_{m}\right)^{2}} \quad y \quad M S E=\frac{1}{n} \sum_{i=1}^{n}\left(y_{i c}-y_{i m}\right)^{2}
$$

Donde " $n$ " es el número de observaciones, yic es el i-ésimo valor calculado, yim es el iésimo valor medido, finalmente $\mathrm{y}_{\mathrm{m}}$ es el valor medio de los valores calculados. La presente investigación se realizó cumpliendo la normativa interna de la Institución Educativa José Gálvez N¹6093 de Chunchuquillo. En este sentido y respetando los valores que todo profesional debe cultivar, los datos se recolectaron con el consentimiento de los estudiantes y padres de familia, comprometiéndonos a guardar la confidencialidad respectiva.

\section{Resultados}

La red neuronal artificial Feed-Forwrad Backpropagation implementada en la Toolbox de Matlab nos facilitó opciones para elegir las funciones de entrenamiento, aprendizaje, desempeño y transferencia, así como también designar el número de capas y el número de neuronas en cada capa, las variables de entrada de la RNA fueron los datos de los estudiantes recolectados mediante el cuestionario, la variable de salida es el promedio anual del estudiante redondeado al entero más cercano. Después de realizar numeroso intentos evaluando los modelos neuronales diseñados teniendo en cuenta la efectividad en la predicción, la Tabla 1 muestra las características del tipo RNA con mayor efectividad de predicción.

\section{Tabla 1}

Características de RNA implementada con mayor efectividad de predicción

\begin{tabular}{ll}
\hline \multicolumn{1}{c}{ Red neuronal artificial } & \multicolumn{1}{c}{ Característica } \\
\hline Tipo de red & Feed-Forwrad Backpropagation \\
Función-entrenamiento & Levenberg Marquardt \\
Función-aprendizaje & Descenso del gradiente \\
Función-desempeño & Error cuadrático medio \\
Función-transferencia & Sigmoidea tangente hiperbólica \\
Neuronas que posee la primera capa oculta & 38 \\
Neuronas que posee la segunda capa oculta & 18 \\
Neuronas que posee la tercera capa oculta & 16 \\
Neuronas que posee la única capa de salida & 1 \\
\hline
\end{tabular}

Nota. El tipo de función y el número de neuronas fueron seleccionados buscando un mejor ajuste del modelo de RNA. 
La arquitectura de esta RNA que se ha diseñado teniendo en cuenta el número de neuronas en cada capa está ilustrado en la Figura 2, se aprecia 38-18-16 neuronas en la primera, segunda y tercera capa oculta, la capa de salida presenta una sola neurona. Input (entrada de la RNA, datos del estudiante). Output (salida de la RNA, promedio anual del estudiante redondeado al entero más cercano).

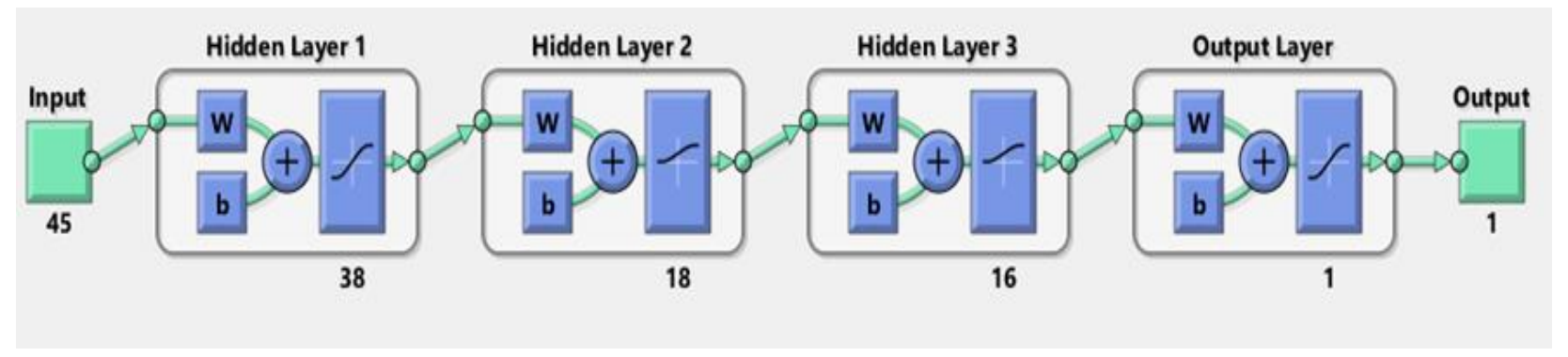

Figura 2. Arquitectura de la RNA implementada en Matlab 2016a. Gráfica obtenida en el Software Matlab 2016a.

Después de realizar distintas pruebas y evaluar cada modelo de RNA mediante el R y MSE (en cada modelo se efectuaron 10 corridas), la red neuronal implementada con las características de la Tabla 1 logró un R y MSE tal como se detalla en la Tabla 2.

\section{Tabla 2}

Coeficiente de correlación y error cuadrático medio

\begin{tabular}{|c|c|c|c|c|c|}
\hline \multirow{3}{*}{$\begin{array}{l}\text { Tipo de Red } \\
\text { Feed-Forwrad } \\
\text { Backpropagation }\end{array}$} & \multicolumn{3}{|c|}{ Coeficiente de correlación } & \multirow{3}{*}{$\begin{array}{l}\text { Coeficiente de } \\
\text { correlación } \\
\text { ponderado } \\
0.967190\end{array}$} & \multirow{3}{*}{$\begin{array}{l}\text { Error } \\
\text { cuadrático } \\
\text { medio } \\
\quad 0.05011\end{array}$} \\
\hline & Entrenamiento & Validación & Prueba & & \\
\hline & 0.989110 & 0.925220 & 0.890730 & & \\
\hline
\end{tabular}

Nota. Valores obtenidos en el Software Matlab 2016a en la octava iteración

La Figura 3 muestra el coeficiente de correlación en cada etapa, permite visualizar la relación que existe entre el promedio obtenido (real) y el promedio esperado (real) del estudiante. Training (entrenamiento), Validation (validación), Test (prueba). 

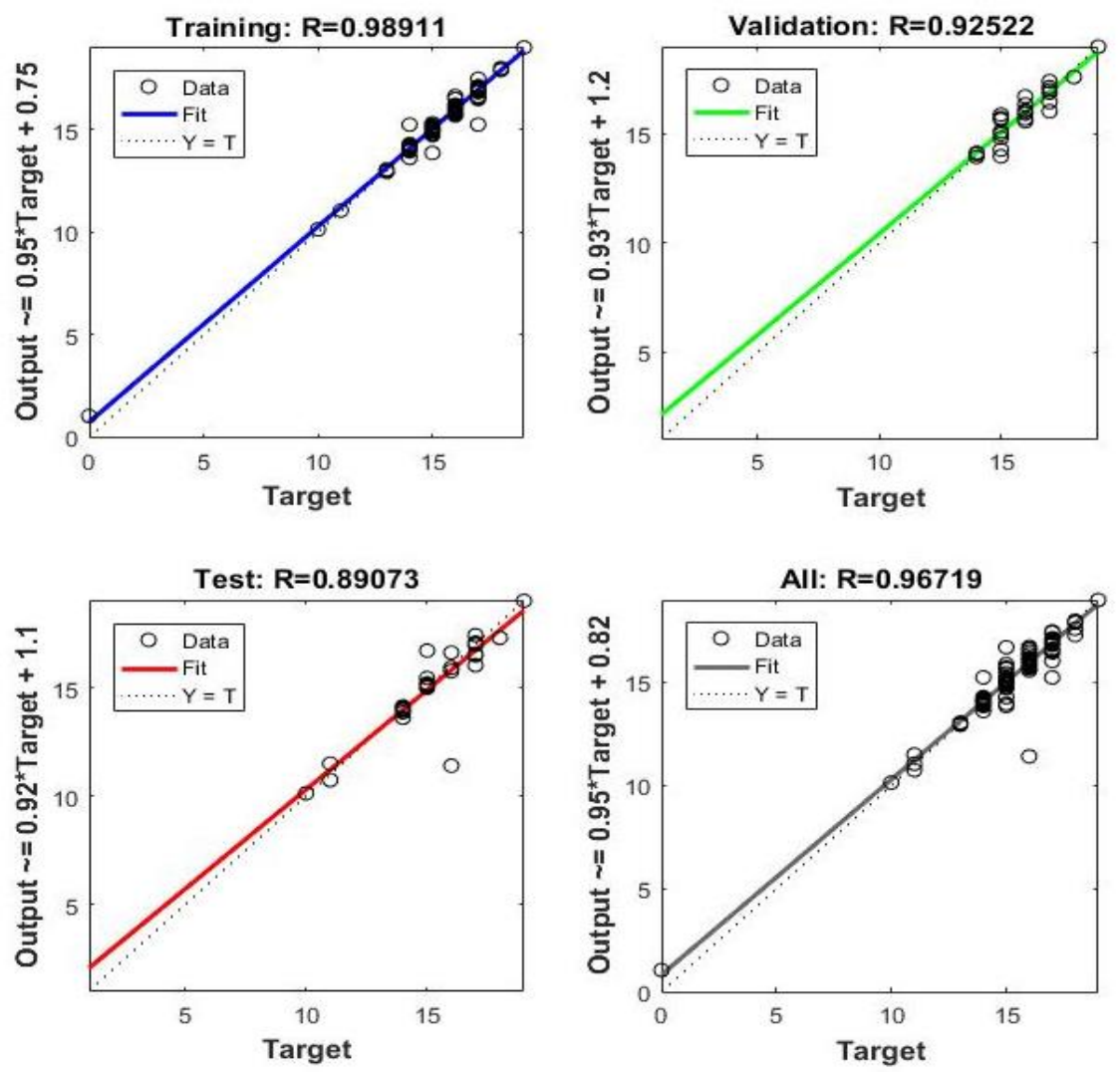

Figura 3. Coeficiente de correlación en cada etapa.

Gráfica obtenida en el Software Matlab 2016a.

En cuanto al error cuadrático medio, la Figura 4 muestra una disminución notable del error mientras la RNA ejecuta cada una de sus procesos. En la segunda iteración se observa un MSE de 0.21385, seguidamente en la octava iteración el MSE disminuye a 0.05011.

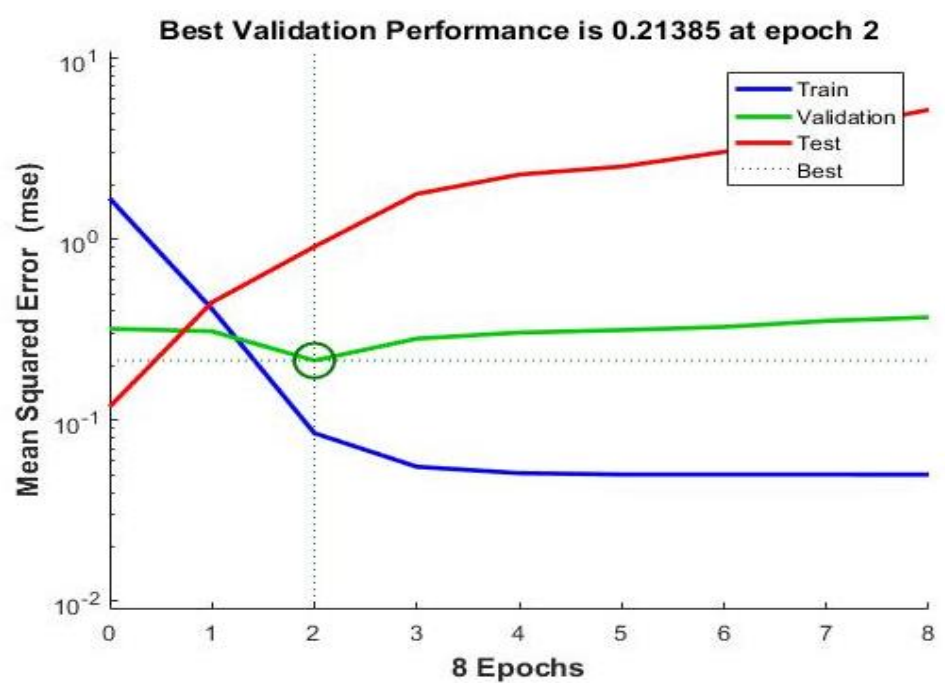

Figura 4. Error cuadrático medio. Gráfica obtenida en el Software Matlab 2016a 
La Tabla 3 muestra la predicción del promedio anual de los 203 estudiantes $(E 001, E 002, \ldots \ldots, E 203)$ que ya cursaron el segundo grado de educación secundaria de la Institución Educativa $\mathrm{N}^{\circ} 16093$ mediante el modelo de red neuronal implementado con las características descritas anteriormente en la Tabla 1 y Tabla 2.

Tabla 3

Predicción del promedio anual de estudiantes del tercer al quinto grado de secundaria

\begin{tabular}{|c|c|c|}
\hline Participantes & $\begin{array}{l}\text { Promedio } \\
\text { final real }\end{array}$ & $\begin{array}{l}\text { Predicción } \\
\text { de la RNA }\end{array}$ \\
\hline E001 & 16 & 16 \\
\hline E002 & 15 & 14 \\
\hline E003 & 16 & 16 \\
\hline E004 & 14 & 14 \\
\hline E005 & 15 & 15 \\
\hline E006 & 15 & 15 \\
\hline E007 & 14 & 14 \\
\hline E008 & 15 & 14 \\
\hline E009 & 15 & 16 \\
\hline E010 & 19 & 19 \\
\hline E011 & 15 & 15 \\
\hline E012 & 16 & 16 \\
\hline E013 & 14 & 14 \\
\hline E014 & 16 & 16 \\
\hline E015 & 15 & 15 \\
\hline E016 & 17 & 17 \\
\hline E017 & 0 & 1 \\
\hline E018 & 17 & 17 \\
\hline E019 & 17 & 17 \\
\hline E020 & 14 & 14 \\
\hline E021 & 17 & 17 \\
\hline E022 & 14 & 14 \\
\hline E023 & 16 & 16 \\
\hline E024 & 16 & 16 \\
\hline E025 & 14 & 14 \\
\hline E026 & 19 & 19 \\
\hline E027 & 10 & 10 \\
\hline E028 & 17 & 16 \\
\hline E029 & 15 & 15 \\
\hline E030 & 17 & 17 \\
\hline E031 & 16 & 16 \\
\hline E032 & 17 & 17 \\
\hline E033 & 17 & 17 \\
\hline E034 & 16 & 16 \\
\hline E035 & 15 & 15 \\
\hline E036 & 18 & 18 \\
\hline E037 & 16 & 16 \\
\hline E038 & 15 & 16 \\
\hline E039 & 17 & 17 \\
\hline E040 & 16 & 16 \\
\hline E041 & 15 & 15 \\
\hline E042 & 15 & 15 \\
\hline
\end{tabular}




\begin{tabular}{|c|c|c|}
\hline E043 & 15 & 17 \\
\hline E044 & 18 & 18 \\
\hline E045 & 18 & 18 \\
\hline E155 & 18 & 18 \\
\hline E156 & 16 & 16 \\
\hline • & - & . \\
\hline • & - & • \\
\hline$\cdot$ & • & • \\
\hline$\cdot$ & $\cdot$ & - \\
\hline$\cdot$ & • & • \\
\hline . & - & • \\
\hline$\cdot$ & • & • \\
\hline$\cdot$ & • & - \\
\hline • & . & • \\
\hline E160 & 17 & 17 \\
\hline E161 & 15 & 15 \\
\hline E162 & 17 & 17 \\
\hline E163 & 14 & 14 \\
\hline E164 & 16 & 16 \\
\hline E165 & 15 & 15 \\
\hline E166 & 16 & 16 \\
\hline E167 & 14 & 14 \\
\hline E168 & 14 & 14 \\
\hline E169 & 14 & 14 \\
\hline E170 & 14 & 14 \\
\hline E171 & 15 & 15 \\
\hline E172 & 14 & 14 \\
\hline E173 & 15 & 15 \\
\hline E174 & 17 & 17 \\
\hline E175 & 14 & 14 \\
\hline E176 & 15 & 15 \\
\hline E177 & 16 & 16 \\
\hline E178 & 14 & 14 \\
\hline E179 & 16 & 16 \\
\hline E180 & 18 & 18 \\
\hline E181 & 17 & 16 \\
\hline E182 & 14 & 15 \\
\hline E183 & 16 & 16 \\
\hline E184 & 13 & 13 \\
\hline E185 & 16 & 16 \\
\hline E186 & 15 & 15 \\
\hline E187 & 16 & 16 \\
\hline E188 & 16 & 16 \\
\hline E189 & 16 & 16 \\
\hline E190 & 17 & 17 \\
\hline E191 & 17 & 17 \\
\hline E192 & 14 & 14 \\
\hline E193 & 17 & 17 \\
\hline E194 & 14 & 14 \\
\hline E195 & 16 & 16 \\
\hline E196 & 15 & 16 \\
\hline E197 & 15 & 15 \\
\hline E198 & 19 & 19 \\
\hline E199 & 11 & 11 \\
\hline
\end{tabular}




\begin{tabular}{lll} 
E200 & 16 & 16 \\
E201 & 15 & 15 \\
E202 & 16 & 17 \\
E203 & 15 & 15 \\
\hline
\end{tabular}

Nota. Se visualiza una efectividad del $88.670 \%$ en la predicción exacta del promedio redondeado al entero más cercano, la efectividad de predicción aumenta a $98.522 \%$ del promedio con una diferencias \pm 1 del mismo.

Garantizada la efectividad en la predicción de la RNA, se aplicó el mismo cuestionario a 18 estudiantes que cursan actualmente el segundo grado de secundaria de la I.E. $\mathrm{N}^{\circ} 16093$ con la finalidad de pronosticar sus promedios anuales correspondientes, la Tabla 4 muestra los resultados de esta predicción.

\section{Tabla 4}

Predicción del promedio de estudiantes del segundo grado de secundaria

\begin{tabular}{lcc}
\hline Participantes & $\begin{array}{c}\text { Promedio final } \\
\text { real }\end{array}$ & $\begin{array}{c}\text { Pronóstico } \\
\text { de la RNA }\end{array}$ \\
\hline E01 & $\ldots \ldots .$. & 15 \\
E02 & $\ldots \ldots .$. & 17 \\
E03 & $\ldots \ldots$. & 10 \\
E04 & $\ldots \ldots$. & 17 \\
E05 & $\ldots \ldots .$. & 17 \\
E06 & $\ldots \ldots$. & 14 \\
E07 & $\ldots \ldots .$. & 17 \\
E08 & $\ldots \ldots .$. & 14 \\
E09 & $\ldots \ldots$. & 16 \\
E10 & $\ldots \ldots .$. & 16 \\
E11 & $\ldots \ldots .$. & 14 \\
E12 & $\ldots \ldots$. & 19 \\
E13 & $\ldots \ldots .$. & 10 \\
E14 & $\ldots \ldots .$. & 16 \\
E15 & $\ldots \ldots$. & 15 \\
E16 & $\ldots \ldots .$. & 17 \\
E17 & $\ldots \ldots .$. & 16 \\
E18 & $\ldots \ldots$. & 17 \\
\hline
\end{tabular}

Nota. Se visualiza la predicción del promedio de 18 estudiantes por medio del modelo de RNA implementado, las variables de entrada de la RNA son recolectadas con el mismo cuestionario aplicado anteriormente a los 203 estudiantes.

En la Figura 5 se visualiza el modelado de los resultados académicos de los estudiantes: Promedio final real (promedio esperado, Tabla 3), promedio producto de la predicción de la RNA implementada (promedio obtenido, Tabla 3) y el pronóstico (Tabla 4) del promedio de 18 estudiantes por medio de la RNA con las condiciones dadas en la Tabla 1 y la Tabla 2. 


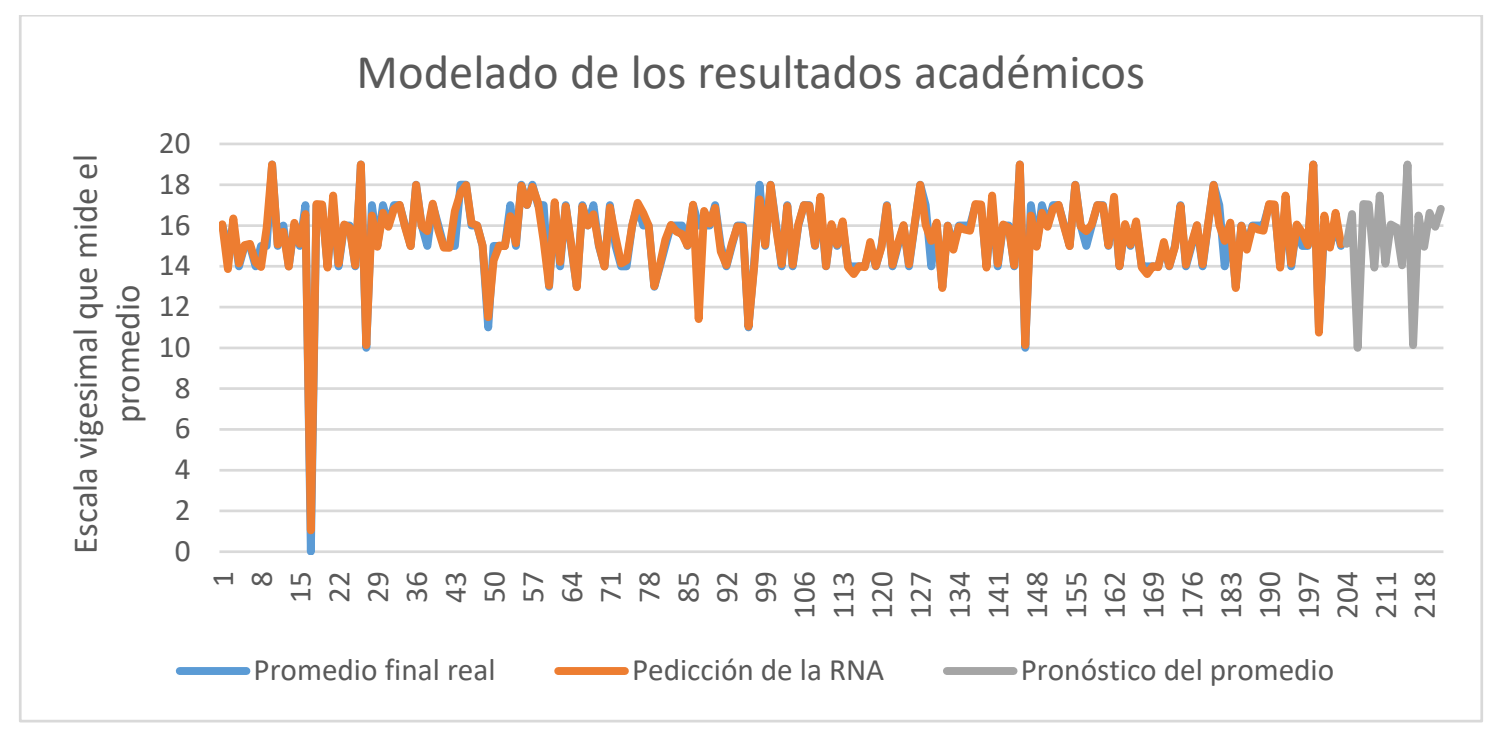

Figura 5. Modelado de resultados académicos

\section{Discusión}

Los resultados obtenidos en esta investigación confirman las afirmaciones dadas por Costa et al. (2017) y Lejía et al. (2010) al resaltar la efectividad en la predicción de resultados académicos que logran las redes neuronales artificiales. Ellos también concuerdan con la efectividad lograda en la predicción al igual que la RNA implementada por Çetinkaya y Baykan (2020) e Incio et al. (2021); sin embargo, se destacan dos situaciones muy importantes y diferentes: 1) ambas investigaciones utilizaron la aplicación nnstart de Matlab logrando los mejores porcentajes de predicción $(78 \%, 75 \%)$ con el algoritmo Levenberg Marquardt; no obstante, en nuestra investigación se utilizó la aplicación nntool de Matlab para diseñar la arquitectura de la RNA, la ventaja de esta última es la flexibilidad que se tiene con respecto a la designación del número de capas, numero de neuronas en cada capa, y las respectivas funciones de activación; por lo que se logró una efectividad en la predicción del $88.670 \%$ y 98.522\% en el promedio anual del estudiante (Tabla 2); 2) Çetinkaya y Baykan (2020) consideraron como una de las variables que le permitía predecir el talento en programación de los estudiantes, a las calificaciones de un curso en Code.org que implementaron de forma presencial, desventaja que nosotros al igual que Incio et al. (2021) tuvimos, puesto que la actual coyuntura sanitaria a causa del Covid-19 lo impide.

La fortaleza del modelo neuronal que se diseñó e implementó en esta investigación radica en la efectividad de predicción que posee, se ha logrado porcentajes predictivos muy altos comparados con las investigaciones citadas en este artículo, este logro se debe al tipo de red que se utilizó, a las funciones de activación escogidas y al número de neuronas que a cada capa se le asignó en las múltiples iteraciones que se realizaron, a pesar de que el tipo de red 
neuronal Feed-forward se caracteriza por la efectividad en la predicción y facilidad en la implementación de su arquitectura, no debemos olvidar las características de la data con que se trabaja; en este sentido concordamos con lo afirmado por Wunderlich y Pehle (2021), Johnson et al. (2021) y Sabir et al (2021) al referirse que los modelos neuronales implementados no deben ser generalizados, es decir cada modelo de RNA debe ser diseñado considerando las características de la población de estudio.

Las redes neuronales artificiales son parte de múltiples herramientas de la inteligencia artificial, que en los últimos años han desempeñado un papel de suma importancia en los múltiples procesos educativos. En este contexto, Menacho (2017) implementa un modelo predictivo con la finalidad de anticiparse al rendimiento académico de estudiantes universitarios, similarmente Martins et al. (2020) implementaron un modelo para anticiparse a la deserción estudiantil, al igual que Lejía et al. (2010), quienes anticiparon los resultados académicos en un centro pre universitario. En general, los avances tecnológicos deben ser involucrados en el sector educación para resolver problemas que este presenta. Además, conocer tempranamente los posibles resultados académicos al final de un determinado periodo en los estudiantes como se observa en la Tabla 4, le otorga al docente la posibilidad de tomar a tiempo las medidas correctivas y de mejora, a fin de revertir estos resultados en el supuesto caso que no sean los mejores, o en su virtud reforzar con éxito el logro de las competencias adquiridas.

Esta investigación aporta conocimiento teórico y práctico en el ámbito educativo: en lo teórico, propicia la investigación multidisciplinaria, involucrando el conocimiento existente de la IA para realizar investigaciones que den solución a problemas que presenta este sector (Moreira et al., 2019); en lo práctico, otorga al docente y a la institución educativa los posibles resultados académicos de la comunidad estudiantil prematuramente, con el objetivo de que los involucrados en el logro de competencias, puedan implementar acciones correctivas y/o de mejora en el proceso de enseñanza y aprendizaje, logrando mejores resultados en el logro de competencias en los estudiantes (López-Aguilar \& Álvarez-Pérez, 2021).

Algunas limitaciones relevantes en el desarrollo de esta investigación, son el acceso a internet de los estudiantes y la deserción estudiantil como consecuencias de la pandemia Covid19. Visto las implicaciones teóricas y prácticas en beneficio de la educación, recomendamos replicar investigaciones que involucren herramientas de la IA en las demás instituciones educativas, teniendo en cuenta las características de la población. Con la finalidad de fortalecer a la comunidad educativa se recomienda realizar investigaciones que involucren la deserción estudiantil, ya que en estos tiempos de pandemia se ha incrementado considerablemente. 


\section{Conclusiones}

Se demuestra que es posible predecir el resultado académico de los estudiantes del segundo grado de la Institución Educativa $N^{\circ} 16093$ en la provincia de Jaén-Perú, el modelo de red neuronal artificial implementado logró una efectividad del $88.670 \%$ en la predicción exacta del promedio redondeado al entero más cercano. Sin embargo, la efectividad aumenta al $98.522 \%$ si se considera una diferencia \pm 1 del promedio.

Las redes neuronales artificiales demuestran ser una herramienta importante en el sector educativo con fines predictivos para lograr una mejora en el logro de resultados; no obstante, los modelos neuronales no deben ser duplicados puesto que cada unidad de estudio tiene sus propias características dentro de un contexto sociocultural en un determinado espacio-tiempo.

\section{Referencias}

Álvarez, J., Lau, R., Pérez, S., \& Leyva, E. C. (2016). Predicción de resultados académicos de estudiantes de informática mediante el uso de redes neuronales. Ingeniare. Revista chilena de ingeniería, 24 (4), 715-727. https://doi.org/10.4067/S071833052016000400015

Barreto, W. \& Picón, R. (2020). Experimental and simulation study of compose panels inelastic behavior using Artificial Neural Networks. Informes de la Construccion, 72(558), 1-10. https://doi.org/10.3989/ic.70957

Çetinkaya, A. \& Baykan, Ö. K. (2020). Prediction of middle school students' programming talent using artificial neural networks. Engineering Science and Technology, an International Journal. https://doi.org/10.1016/j.jestch.2020.07.005

Comella, A., Casas-Baroy, J.-C., Comella-Company, A., Galbany-Estragués, P., Pujol, R. \& Marc-Amengual, J.-M. (2021). Burnout and academic performance: Effect of the combination of remunerated jobs and starting university degree studies. Retos, 41, 844853. https://doi.org/10.47197/RETOS.V41I0.85971

Costa, E. B., Fonseca, B., Santana, M. A., de Araújo, F. F., \& Rego, J. (2017). Evaluating the effectiveness of educational data mining techniques for early prediction of students' academic failure in introductory programming courses. Computers in Human Behavior, 73, 247-256. https://doi.org/10.1016/j.chb.2017.01.047

Criollo, M., Romero, M., \& Fontaines-Ruiz, T. (2017). Autoeficacia para el aprendizaje de la investigación en estudiantes universitarios. Psicología Educativa, 23 (1), 63-72. https://doi.org/10.1016/j.pse.2016.09.002 
da Silva, A. X., de Oliveira, S. C., \& de Araújo, R. F. G. (2020). Propuesta de prototipo de aplicación de Android para diagnósticos de enfermería utilizando redes neuronales artificiales. Revista Cubana de Enfermería, 36 (2), Article 2. http://revenfermeria.sld.cu/index.php/enf/article/view/3252

Díaz-Muñoz, G. (2020). Metodología del estudio piloto. Revista chilena de radiología, 26 (3), 100-104. https://doi.org/10.4067/S0717-93082020000300100

Echeverría-Ramírez, J. A., \& Mazzitelli, C. (2021). A study of the perception of the institutional factors that influence the academic performance of students of the Distance State University of Costa Rica. Revista Electrónica Educare, 25 (2). https://doi.org/10.15359/ree.25-2.18

Ferraces, M. J., Moledo, M. L., Otero, A. G. \& Santos, M. A. (2021). Students' mediator variables in the relationship between family involvement and academic performance: Effects of the styles of involvement. Psicologia Educativa, 27 (1), 85-92. https://doi.org/10.5093/PSED2020A19

Figueroa-Garcia, E., Farias-Cervantes, V. S., Segura-Castruita, M., Andrade-Gonzalez, I., Montero-Cortés, M. I., \& Chávez-Rodríguez, A. M. (2021). Using artificial neural networks in prediction of the drying process of foods that are rich in sugars. Revista Mexicana de Ingeniera Quimica, $20 \quad$ (1), 161-171. https://doi.org/10.24275/rmiq/Sim1403

Foster, R. C. (2020). A generalized framework for classical test theory. Journal of Mathematical Psychology, 96. https://doi.org/10.1016/j.jmp.2020.102330

Foster, R. C. (2021). KR20 and KR21 for Some Nondichotomous Data (It's Not Just Cronbach's Alpha). Educational and Psychological Measurement. https://doi.org/10.1177/0013164421992535

González-García, N., Sánchez-García, A. B., Nieto-Librero, A. B., \& Galindo-Villardón, M. P. (2019). Actitud y enfoques de aprendizaje en el estudio de la Didáctica General. Una visión multivariante. Revista de Psicodidáctica, 24 (2), 154-162. https://doi.org/10.1016/j.psicod.2019.02.002

González-Velasco, C., Feito-Ruiz, I., González-Fernández, M., Álvarez-Arenal, J.-L. \& Sarmiento-Alonso, N. (2021). Does the teaching-learning model based on the flipped classroom improve academic results of students at different educational levels? Revista Complutense de Educacion, 32 (1), 27-39. https://doi.org/10.5209/RCED.67851 
Goyes-Peñafiel, P. \& Hernandez-Rojas, A. (2021). Double landslide susceptibility assessment based on artificial neural networks and weights of evidence. Boletin de Geologia, 43 (1), 173-191. https://doi.org/10.18273/revbol.v43n1-2021009

Hernández, R. M. (2018). La estrategia didáctica frente a los estilos de aprendizaje en la $\begin{array}{llll}\text { educación } & \text { superior. } & \text { Educación } & \text { Médica, }\end{array}$ https://doi.org/10.1016/j.edumed.2017.10.034

Incio, F. A. \& Capuñay, D. L. (2020). Liderazgo directivo y desempeño docente en instituciones educativas particulares. Revista Científica Epistemia, 4 (3), 119-128. https://doi.org/10.26495/re.v4i3.1422

Incio, F. A., Capuñay, D. L., Estela, R. O., Delgado, J. A. \& Vergara, S. E. (2021). Diseño e implementación de una red neuronal artificial para predecir el rendimiento académico en estudiantes de Ingeniería Civil de la UNIFSLB. Revista Veritas et Scientia, 10 (1), 107-117. https://doi.org/10.47796/ves.v10i1.464

International Business Machines Corporation. (2021). Conceptos básicos de ayuda de CRISPDM. Conceptos básicos de ayuda de CRISP-DM. https://prod.ibmdocs-production-dal6099123ce774e592a519d7c33db8265e-0000.us-

south.containers.appdomain.cloud/docs/es/spss-modeler/SaaS?topic=dm-crisp-helpoverview

Johnson, K. B., Ferguson, D. H., Tempke, R. S. \& Nix, A. C. (2021). Application of a Convolutional Neural Network for Wave Mode Identification in a Rotating Detonation Combustor Using High-Speed Imaging. Journal of Thermal Science and Engineering Applications, 13 (6). https://doi.org/10.1115/1.4049868

Lejía, C.-T., Ng, L.-N., Hassan, M. D., Goh, W.-W., Ley, C.-Y. \& Ismail, N. (2010). Predicting pre-university students' mathematics achievement. 8, 299-306. https://doi.org/10.1016/j.sbspro.2010.12.041

López-Aguilar, D. \& Álvarez-Pérez, P. R. (2021). Modelo predictivo PLS-SEM sobre intención de abandono académico universitario durante la COVID-19. Revista Complutense de Educación, 32 (3), 451-461. https://doi.org/10.5209/rced.70507

Martins, M. P. G., Migueis, V. L., Fonseca, D. S. B. \& Gouveia, P. D. F. (2020). Prediction of academic dropout in a higher education institution using data mining. RISTI - Revista Iberica de Sistemas e Tecnologias de Informacao, 2020 (E28), 188-203.

Menacho, C. H. (2017). Predicción del rendimiento académico aplicando técnicas de minería de datos. Anales Científicos, 78 (1), 26-33. https://doi.org/10.21704/ac.v78i1.811 
Moreira, F., Ferreira, M. J., Pereira, C. S., Gomes, A. S., Collazos, C. \& Escudero, D. F. (2019). ECLECTIC as a learning ecosystem for higher education disruption. Universal Access in the Information Society, 18 (3), 615-631. https://doi.org/10.1007/s10209-019-00682$\mathbf{X}$

Olascoaga, A. C. (2020). Curso de docencia para residentes: Evaluación de un programa. Educación Médica, 21 (3), 187-192. https://doi.org/10.1016/j.edumed.2018.07.010

Pal, J. \& Chakrabarty, D. (2020). Assessment of artificial neural network models based on the simulation of groundwater contaminant transport. Hydrogeology Journal, 28 (6), 20392055. https://doi.org/10.1007/s10040-020-02180-4

Pizarro, S., Santillán, G., Vilela, J. \& Hildebrandt, A. (2021). Factors related to academic performance in Veterinary Medicine students. Revista de Investigaciones Veterinarias del Peru, 32 (1). https://doi.org/10.15381/RIVEP.V32I1.19509

Porras, M. M. \& Ortega, F. H. (2021). Procrastination, test anxiety and academic performance on university students. Interdisciplinaria, $38 \quad$ (2), 243-258. https://doi.org/10.16888/INTERD.2021.38.2.16

Ramos, R. T. \& Gómez, N. N. (2021). The influence of the teacher on the motivation, learning strategies, critical thinking and academic performance of high school students in Physical Education. Psychology, Society and Education, 11 (1), 137-150. https://doi.org/10.25115/psye.v11i1.2230

Rodríguez, D. D., Ordoñez, R. E. \& Hidalgo-Villota, M. E. (2021). Determinantes del rendimiento académico de la educación media en el departamento de Nariño, Colombia. Lecturas de Economía, 94, 87-126. https://doi.org/10.17533/udea.le.n94a341834

Rodríguez, H. E. D., Castro, M. M. S. \& Rosales, M. A. C. (2021). Financial performance and administrative practices in Mexican microenterprises: An analysis with artificial neural $\begin{array}{lllll}\text { networks. } & \text { Contaduria } & y & \text { Administracion, } & 64\end{array}$ https://doi.org/10.22201/FCA.24488410E.2018.1622

Sabir, Z., Nisar, K., Raja, M. A. Z., Ibrahim, A. A. B. A., Rodrigues, J. J. P. C., Al-Basyouni, K. S., Mahmoud, S. R. \& Rawat, D. B. (2021). Design of Morlet wavelet neural network for solving the higher order singular nonlinear differential equations. Alexandria Engineering Journal, 60 (6), 5935-5947. https://doi.org/10.1016/j.aej.2021.04.001

Sagredo, A. V., Etchepare, G. C., Mendizabal, E. A. \& Wilson, C. P. (2021). Academic performance and its relationship with socioemotional variables in chilean students from vulnerable contexts. Educacion XX1, $24 \quad$ (2), 375-398. https://doi.org/10.5944/educxx1.28269 
Şenyurt, M., Ercanl1, İ., Günlü, A., Bolat, F. \& Bulut, S. (2020). Artificial neural network models for predicting relationships between diameter at breast height and stump diameter: Crimean pine stands at ÇAKÜ forest. Bosque, 41 (1), 25-34. https://doi.org/10.4067/S0717-92002020000100025

Sosa, M., Ortiz, E. \& Cabello, A. (2021). Impact of social lags on the number of deaths and confirmed cases by COVID-19 in Mexico: Artificial neural network analysis employing municipal information. Contaduria y Administracion, 65 (5). https://doi.org/10.22201/fca.24488410e.2020.3020

Wingerter, D. G., de Oliveira Santos, E. G. \& Barbosa, I. R. (2020). The use of artificial neural networks to classify the social vulnerability of municipalities in Rio Grande do Norte State, Brazil. Cadernos de Saude Publica, 36 (8). https://doi.org/10.1590/0102311 X00038319

Wunderlich, T. C. \& Pehle, C. (2021). Event-based backpropagation can compute exact gradients for spiking neural networks. Scientific Reports, 11 (1). https://doi.org/10.1038/s41598-021-91786-z 